\title{
Modal response and frequency shift of the cantilever in a noncontact atomic force microscope
}

\author{
Wei L. Wang ${ }^{\text {a) }}$ and S. Jack Hu \\ The University of Michigan, Ann Arbor, Michigan 48109
}

(Received 29 March 2005; accepted 22 September 2005; published online 26 October 2005)

\begin{abstract}
The force-sensing cantilever in a noncontact atomic force microscope is a continuous system with infinite number of eigenmodes. Although the frequently used point mass model was found sufficient in many cases, its conditions for validity and the insights on how higher eigen-modes could affect the selection of operation parameters were not established. In this letter, we formulate the cantilever motion using modal response analysis, a powerful means enabling an efficient numerical solution and a first order analytical solution. The origins and impacts of the higher eigenfrequency oscillation are then investigated, which sheds lights on achieving optimal imaging conditions. () 2005 American Institute of Physics. [DOI: 10.1063/1.2123391]
\end{abstract}

A frequency modulation version of the dynamic atomic force microscope, ${ }^{1}$ the so called noncontact atomic force microscope (nc-AFM), has been proven to be a powerful tool in producing atomic resolution images on various surfaces including conducting, nonconducting, and even reactive ones. However, the search for the true atomic resolution had not been straightforward. Most times, the microscope design and operating parameters for achieving optimal imaging conditions were identified empirically. It was not well understood why some parameters yielded good images while others did not. To understand the imaging mechanism, some important modeling work has been done. Among them, the frequency shift has been calculated with perturbation theory, ${ }^{2-4}$ variational methods, ${ }^{5,6}$ or numerical integration of Newtonian equation of motion. ${ }^{3,4}$ All these studies assumed that the multiple degrees of freedom (DOF) cantilever could be sufficiently represented by a single DOF point mass and the higher eigenmodes of the continuous system were neglected. Although such point mass assumption was found consistent with experimental results in many cases, the conditions for validity of the model is not clear and the insights on how these higher eigenfrequencies could affect the cantilever motion and operation parameter selection were not established. In other studies ${ }^{7-13}$ on the dynamic AFM, continuous cantilever model was used. However they either focused only on the tapping mode scenario where tip-surface interaction is relatively strong, or treated the tip-surface interaction as a nonlinear boundary condition, which made an efficient direct modal analysis inapplicable. In this letter, we formulate the motion of the cantilever using modal response analysis, which turns out to be powerful in accounting higher eigenmodes, dealing with weak perturbation, and deriving analytical solutions.

Modeling the cantilever as an Euler-Bernoulli beam, its motion can be described by

$$
-\frac{\partial^{2}}{\partial x^{2}}\left[E I(x) \frac{\partial^{2} y(x, t)}{\partial x^{2}}\right]+f(x, t)=m(x) \frac{\partial^{2} y(x, t)}{\partial t^{2}},
$$

and the boundary conditions are $y(0, t)=0, y^{\prime}(0, t)=0$, $y^{\prime \prime}(L, t)=0$, and $y^{\prime \prime \prime}(L, t)=0$, where $L$ is the length of the can-

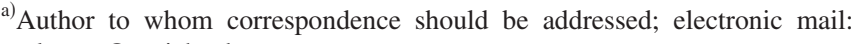
wlwang@umich.edu
}

tilever whose left end is fixed at the origin. $I(x)$ and $m(x)$ are the moment of inertia and mass density. In general, the term $f(x, t)$ is a time dependent distributed force, representing the tip surface interaction, the damping force, and the controlled feedback driving force. For an nc-AFM operating in an UHV, the latter two components are very small and tend to cancel each other, thus neglected. ${ }^{4}$ The tip-surface interaction force distributed locally at the right end of the cantilever can be treated as an excitation ${ }^{9,10}$ instead of boundary condition $^{7,8}$ in order to directly apply modal analysis. Such treatment is justified by writing

$$
f(x, t)=F(t) \delta\left(x-L^{-}\right),
$$

where $F(t)$ is the "concentrated" tip-surface interaction force and $\delta(x)$ Dirac's delta function. Position $L^{-}$approaches the free end of the cantilever $L$ from the left side. ${ }^{14}$ The motion of the linear system (cantilever) can then be transformed into modal coordinates in which the generalized force is given by

$$
\begin{aligned}
Q_{r}(t) & =\int_{0}^{L} F(t) \delta\left(x-L^{-}\right) \psi_{r}(x) d x=F(t) \psi_{r}\left(L^{-}\right) \\
& =F(t) \psi_{r}(L),
\end{aligned}
$$

where $\psi_{r}(x)$ is the $r$ th eigenfunction. The deflection at $x=L$ can be found from

$$
\frac{1}{\psi_{r}^{2}(L)} \ddot{y}_{r}(t)+\frac{\omega_{r}^{2}}{\psi_{r}^{2}(L)} y_{r}(t)=F(t) .
$$

The effective mass and effective stiffness of the $r$ th mode can thus be defined as $M_{r}=1 / \psi_{r}^{2}(L), k_{r}=\omega_{r}^{2} / \psi_{r}^{2}(L)$. Applying time response equations, ${ }^{15}$ we obtain the displacement of the cantilever at $x=L$,

$$
\begin{aligned}
y(t)= & \sum_{r=0}^{\infty}\left[\frac{\psi_{r}^{2}(L)}{\omega_{r}} \int_{0}^{t} \sin \omega_{r}(t-\tau) F(\tau) d \tau+y_{r}(0) \cos \omega_{r} t\right. \\
& \left.+\frac{\dot{y}_{r}(0)}{\omega_{r}} \sin \omega_{r} t\right] .
\end{aligned}
$$

The convolution term in the equation, also written as $\Delta y(t)$ is the deviation due to the weak perturbation $F(\tau)$, which is the only term that needs to be numerically calculated since the responses due to the initial conditions are 


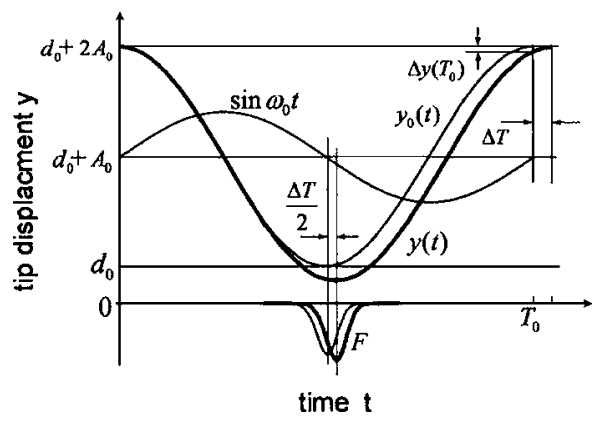

FIG. 1. First order approximation of cantilever motion $y(t)$ within one period illustrates the deviation of the actual motion from the unperturbed motion $y_{0}(t)$ and the origin of the frequency shift.

readily given in analytical forms. Such separation of responses is important in establishing an efficient and robust numerical simulation, especially in the case of nc-AFM where the response due to the tip-surface interaction is much smaller than that originated from the initial free oscillation.

For a first order analytical solution, neglecting the terms of $r>0$ and calculating the deviation term in Eq. (2) at $T_{0}$ (the free oscillation period) yield

$$
\Delta y\left(T_{0}\right)=-\frac{\psi_{0}^{2}(L)}{\omega_{0}} \int_{0}^{T_{0}} F(\tau) \sin \omega_{0} \tau \cdot d \tau,
$$

where the tip-surface interaction force is deflection (thus time) dependent. For a free oscillation subjected to the initial conditions $y_{0}(0)=A_{0}$ and $\dot{y}_{0}(0)=0$, the displacement is $y_{0}$ $=d_{0}+A_{0}+A_{0} \cos \omega_{0} \tau$ as illustrated in Fig. 1. In the presence of a weak interaction force, if the perturbation Hamiltonian is much smaller than the unperturbed Hamiltonian, we could assume that the perturbed displacement remains approximately harmonic but with slightly changed amplitude and frequency: ${ }^{2-4} y=d_{0}+2 A_{0}-A+A \cos \omega \tau$, where $A$ and $\omega$ are unknown. Expand $F(\tau)=F[y(\tau)]$ with respect to $A$ and $\omega$ at $y_{0}$, and neglect higher order terms, we have

$$
\begin{aligned}
F(y) \approx & F\left(y_{0}\right)+\frac{\partial F\left(y_{0}\right)}{\partial y_{0}}\left(-A_{0} \sin \theta_{0} \tau\right) \tau \cdot \Delta \omega \\
& +\frac{\partial F\left(y_{0}\right)}{\partial y_{0}}\left(\cos \omega_{0} \tau-1\right) \cdot \Delta A .
\end{aligned}
$$

On the right-hand side, the first and the third terms are symmetric about $t=T_{0} / 2$, thus vanish in the convolution of Eq. (3). As a result

$$
\Delta y\left(T_{0}\right)=\frac{A_{0} \psi_{0}^{2}(L) \Delta f}{f_{0}} \int_{0}^{1 / f_{0}} \frac{\partial F\left(y_{0}\right)}{\partial y_{0}} \sin ^{2} 2 \pi f_{0} \tau \cdot \tau \cdot d \tau .
$$

On the other hand, at $t=T_{0}$, Taylor's expansion of $y=d_{0}+2 A_{0}-A+A \cos \omega t$ with respect to $A$ and $\omega$ to the second order yields

$$
\Delta y\left(T_{0}\right) \approx-2 \pi^{2} A_{0} \frac{\Delta f^{2}}{f_{0}^{2}} .
$$

Equating this equation with Eq. (4) associates the frequency shift with the generalized force and gives a solution to the frequency shift

$$
\Delta f=-\frac{Y_{0}^{2}(L) f_{0}}{2 \pi^{2}} \int_{0}^{1 / f_{0}} \frac{\partial F\left(y_{0}\right)}{\partial y_{0}} \sin ^{2} 2 \pi f_{0} \tau \cdot \tau \cdot d \tau .
$$

When the gradient $\partial F\left(y_{0}\right) / \partial y_{0}$ is virtually constant, for example, in small amplitude nc-AFMs, using

$$
\begin{aligned}
& k_{0}=\frac{4 \pi^{2} f_{0}^{2}}{\psi_{0}^{2}(L)}, \\
& \int_{0}^{2 \pi} \sin ^{2} \theta \cdot \theta \cdot d \theta=\pi^{2} .
\end{aligned}
$$

Equation (6) is reduced to $\Delta f=-\partial F\left(y_{0}\right) / \partial y_{0} \cdot f_{0} / 2 k_{0}$, which is equivalent to the frequency change given in the first work on frequency modulated AFM by Albrecht. ${ }^{1}$ In the general case where $\partial F\left(y_{0}\right) / \partial y_{0}$ is not constant. The term $\partial F\left(y_{0}\right) / \partial y_{0}$ in Eq. (6) can be replaced by $F\left(y_{0}\right)$ using integration by parts, which transforms Eq. (6) to a form identical to that derived in previous studies using perturbation theory ${ }^{2}$ and variational methods. ${ }^{6}$

Beyond the first order approximation, we are more interested in understanding the origins and impacts of higher eigenmodes in an nc-AFM. Inspecting Eq. (2), we could immediately see that the higher eigenfrequencies become prominent in three conditions: (1) The ratio of $\psi_{r}^{2}(L) / \omega_{r}$ is larger for $r \geqslant 1$; (2) The higher frequency components of the interaction force $F(\tau)$ increases; (3) One or more of the higher eigenfrequencies $\omega_{r}$ get close to an integer multiplication of the interaction force frequency $\omega$, in which resonance occurs.

The ratio $\psi_{r}^{2}(L) / \omega_{r}$, also written as $\omega_{r} / k_{r}$, the ratio of the eigenfrequency and the corresponding effective stiffness is proportional to the induced oscillation at that eigenfrequency. Ideally, one would want a large $\omega_{0} / k_{0}$ thus a large frequency shift to increase the nc-AFM sensitivity but a small $\omega_{r} / k_{r}$ for $r \geqslant 1$ to suppress the higher modes. However, in reality, it is generally not feasible to manipulate those values independently. Instead, they scale up and down together. That means if no noticeable higher modes are allowed, the frequency shift needs to be small as well. This explains why the amount of frequency shift used in the successful nc-AFM experiments cannot be large and the stiffness of cantilever must be relatively high (compared to that of a contact mode AFM). If the earlier frequency-stiffness ratio becomes large, higher eigenmodes will be induced. It was reported in previous study $^{16}$ on the tapping mode AFM that these oscillations are much smaller than the amplitude of the fundamental eigenfrequency and thus can be ignored. Simulation in Fig. 2, however, shows that, in a nc-AFM, it does not take a large amplitude at higher eigenfrequency to generate significant impact on the short range interaction force-a small one will do.

With the minor higher eigenmode oscillations superimposed to that of $f_{0}$, the total vibration amplitude is virtually unchanged but the lowest tip-surface distance is periodically modified due to the phase differences between the higher eigen-frequencies and the fundamental eigenfrequency. Although the modification is small, in the tip-surface interaction force field that has a short decay length, the resulted interaction force modulation is significant, i.e., the peak interaction force varies considerably. The direct consequence of such variation is that mapping from frequency shift to peak force becomes not very meaningful since the peak force quickly varies. In addition, according to previous experiments, the force range for obtaining good images is very narrow. A varying interaction forces would reduce the sensi- 


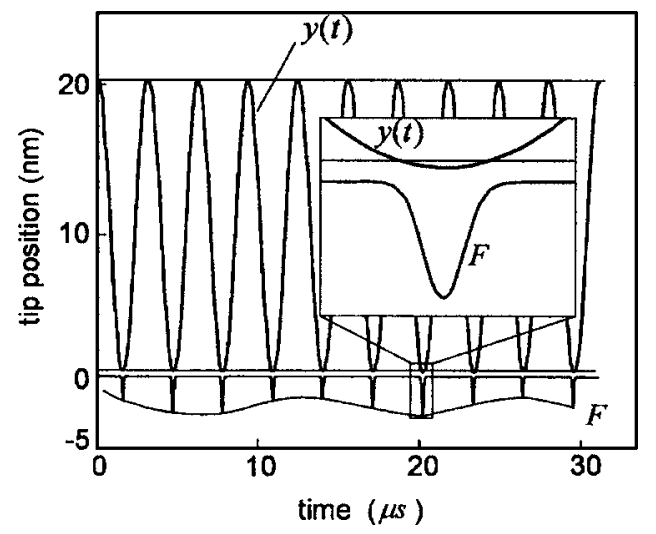

FIG. 2. Simulated cantilever motion $y(t)$ under the tip-surface interaction $F$. Four higher eigenfrequencies are included in the calculation and a uniform cantilever with $f_{0}=161 \mathrm{kHz}, k_{0}=2 \mathrm{~N} / \mathrm{m}$ is used. The short range interaction is of a Lennard-Jones form with equilibrium distance $\sigma=2.5 \AA$ and bonding energy $E_{\text {bond }}=2 \mathrm{eV}$.

tivity and prone to trigger sudden modification to the tip structure. Giessibl ${ }^{2}$ has pointed out that instable imaging condition due to "jump to contact" (JTC) can be avoided if $k_{0} A_{0}>-F$. In Fig. 2, although $k_{0} A_{0}(\sim 20 \mathrm{nN}) \gg-F(\sim 2 \mathrm{nN})$, i.e., JTC did not occur, instable imaging conditions still exist as stated above. Therefore, avoiding JTC does not guarantee stable imaging conditions; it additionally requires the frequency-stiffness ratios be low enough to suppress the higher eigenmodes.

On the other hand, no higher eigenmodes would be induced if $F$ is free of higher eigenfrequency components. In the frequency domain, the dominant peak of $F$ is at the cantilever oscillation frequency $\omega$. However, with the decrease of tip-surface distance, in the time domain, $F$ gets closer to a pulse functions $F \delta\left(t_{n}\right)$ whose frequency content is uniformly across all frequencies. Such increase of higher frequency contents stimulates higher eigenmode oscillation of the cantilever and affects the frequency shift. Simulations based on Eq. (2) shows that, in both attractive and repulsive regime, taking into account higher eigenfrequencies predicts a frequency shift with smaller amplitude than the calculation based on the point mass assumption.

The third origin, higher eigenmode resonance could occur even if the first two conditions are not met. Simulation shows that the effect of such resonance is similar to the modulation shown in Fig. 2 but with an extended time for the amplitude to grow, which could trigger sudden modification to the tip structure.

In short, higher eigenmodes when present have significant effects in a nc-AFM, therefore, limit the design and operating parameters. These parameters should be properly chosen to avoid the above three origins of higher eigenmodes in order to achieve stable imaging conditions and high sensitivity.

The authors wish to thank N. Perkins for stimulating discussions.

${ }^{1}$ T. R. Albrecht, P. Grütter, D. Horne, and D. Rugar, J. Appl. Phys. 69, 668 (1991).

${ }^{2}$ F. J. Giessibl, Phys. Rev. B 56, 16010 (1997).

${ }^{3}$ N. Sasaki and T. Masaru, Appl. Surf. Sci. 140, 339 (1999).

${ }^{4}$ H. Hölscher, U. D. Schwarz, and R. Wiesendanger, Appl. Surf. Sci. 140, 344 (1999).

${ }^{5}$ A. I. Livshits and A. Shluger, Phys. Rev. B 59, 2436 (1999).

${ }^{6}$ U. Dürig, Appl. Phys. Lett. 75, 433 (1999).

${ }^{7}$ J. A. Turner, J. Appl. Phys. 82, 966 (1997).

${ }^{8}$ K. Wolf and O. J. Gottlieb, J. Appl. Phys. 91, 4701 (2002).

${ }^{9}$ R. W. Stark and W. M. Heckl, Surf. Sci. 457, 219 (2000).

${ }^{10}$ R. W. Stark, Nanotechnology 15, 347 (2004).

${ }^{11}$ M. Stark, R. W. Stark, M. Heckl, and R. Guckenberger, Appl. Phys. Lett. 77, 3293 (2000)

${ }^{12}$ O. Sahin and A. Atalar, Appl. Phys. Lett. 79, 4455 (2001).

${ }^{13}$ R. Hillenbrand, M. Stark, and R. Guckenberger, Appl. Phys. Lett. 76, 3478 (2000).

${ }^{14}$ Similar treatment is seen at L. Meirovitch, Fundamentals of Vibrations (McGraw-Hill, New York, 2001).

${ }^{15}$ L. Meirovitch, Elements of Vibration Analysis (McGraw-Hill, New York, 1986).

${ }^{16}$ T. R. Rodríguez and R. García, Appl. Phys. Lett. 80, 1646 (2002). 